\title{
Linking Person and Context in the Daily Stress Process
}

\author{
Avshalom Caspi, Niall Bolger, and John Eckenrode \\ Cornell University
}

\begin{abstract}
In this study we combined daily diary data with interview data to investigate individual differences in the impact of stressful daily events on mood. Using a sample of 96 women in an urban community, we examined perceived neighborhood quality and major life events as possibly potentiating the effects of stressful daily events, and we viewed social supports as potentially buffering this daily process. Results confirmed that the presence of chronic ecologic stress (ncighborhood perceptions) exacerbated the immediate effects of stressful daily events on mood and also increased the likelihood of enduring effects of daily stressors on next day's mood. Contrary to expectations, previous exposure to major life events decreased the impact of stressful daily events. The availability of social supports, although not buffering the impact of stressful daily events on mood, did mitigate the enduring effects of these events on next day's mood. This study also presents a method for analyzing daily time-series data, while correcting for potential problems of autocorrelated error terms. As such, this study represents a significant advance over previous analytic approaches to time-series data in the study of the stress process.
\end{abstract}

Some days everything seems to go wrong, and by day's end, minor difficulties find their outlet in rotten moods. This basic feature of daily life is increasingly attracting the attention of researchers concerned with assessing the relation of environmental stressors to psychological distress. The recent emphasis on daily events is due, in part, to the extensive criticism directed at traditional approaches to the study of environmental stress. The major life events approach assumes that there is a temporal association between an increase in objective events such as divorce and unemployment, which disrupt an individual's usual activities, and the onset of psychological distress. This approach has been criticized from several fronts. The psychometric properties of life-event questionnaires are seldom adequate (Neugebauer, 1981); measures of undesirable life events and outcome criteria may be operationally confounded (Thoits, 1981); and the predictive validity of life event measures is, at best, moderate (Rabkin \& Struening, 1976).

In contrast to the major life events approach, stress researchers are increasingly attending to the ongoing stressors and strains that characterize everyday life and their relation to psychological functioning. Daily stressors range from the ordinary troubles of family life (e.g., the demands of children) to conflicts in the workplace (e.g., work overload) and aspects of the physi-

This research was supported by Grant HS03029 from the National Center for Health Services Research and by Grant 82081000 from the William T. Grant Foundation.

The authors contributed equally to this article. Avshalom Caspi is now at the Department of Psychology, Harvard University. Niall Bolger is now at the Institute for Social Research, University of Michigan.

We gratefully acknowledge the valuable comments of Urie Bronfenbrenner, Steve Cornelius, Geraldine Downey, Glen Elder, Chuck Henderson, Ron Kessler, Dave Riley, and Elaine Walker on earlier drafts of this article.

Correspondence concerning this article should be addressed to John Eckenrode, Department of Human Development and Family Studies, MVR Hall, Cornell University, Ithaca, New York 14853. cal environment (e.g., rush hour traffic). Perhaps because daily stressors appear so ordinary and mundane, their critical contribution to psychological distress and health outcomes has been underemphasized. McLean (1976, p. 298), however, has argued, on the basis of clinical data, that "microstressors, acting cumulatively, and in the relative absence of compensatory experiences, can be potent sources of stress." Lazarus and his colleagues have emphasized the mediational role of relatively minor stresses in the prediction of adaptational outcomes: "It is these day-to-day events that ultimately should have proximal significance for health outcomes and whose cumulative impact, therefore, should also be assessed" (Kanner, Coyne, Schaefer, \& Lazarus, 1981, p. 4; also see DeLongis, Coyne, Dakof, Folkman, \& Lazarus, 1982; Lazarus \& Cohen, 1977). Thus, two functions have been attributed to daily stressors. First, they have been conceptualized as making an independent contribution to psychological well-being. Second, they are thought to mediate the effects of more major forms of stressful life experiences.

The increased emphasis on the nature of stressful daily events and their relation to psychological and physical health outcomes raises new methodological as well as substantive issues. In the present study, diary data, collected over a period of one month from a sample of women in an urban setting, are used to examine the effects of stressful daily events on mood. The major substantive issue that is addressed concerns individual differences in effects of stressful daily events in lowering mood. The major methodological concern is with the appropriate statistical strategies for analyzing these types of time-series data. The present study, and its dual foci, represent an extension of previous research by Eckenrode (1984) based on the same set of data.

\section{Interindividual Variability in the Daily Stress Process}

Interindividual variability in the relation of stressful daily events to mood has not been examined in previous research. Such variability calls for more careful consideration of factors 
that may differentiate vulnerable persons from resilient ones. Most previous studies of the relation of daily stressors to psychological outcomes (e.g., Eckenrode, 1984; Kanner et al., 1981; Rehm, 1978; Stone \& Neale, 1984) either did not consider such individual differences or simply noted the presence of interindividual variability without explicating its nature (e.g., Lewinsohn \& Talkington, 1979).

Evidence from studies of drastic life change (e.g., Elder, Caspi, \& Van Nguyen, 1986), however, suggests that stressful times are not necessarily bad times, and the causal sequence from drastic life change to psychological distress may be conditioned by a host of individual and contextual factors. Do similar factors moderate the relation of daily events to mood? In the present study we examined three factors: the potentiating effects of chronic ecological conditions and major life events and the buffering effects of informal social supports.

\section{Chronic Ecological Stress}

Epidemiological studies suggest that community characteristics play an important role in the genesis of mental illness and behavior disorders (e.g., Leighton, 1974; Rutter \& Madge, 1976; Srole, Langner, Michael, Oples, \& Rennie, 1962). Evidence for the operation of community influences also appears in relation to child abuse. For example, Garbarino and Sherman (1980) were able to develop, on the basis of interviews with key informants, community profiles that support the concept of differential neighborhood risk for child abuse. Within the field of environmental psychology, a substantial amount of evidence has been marshaled in support of the adverse effect of chronic conditions such as noise (Glass \& Singer, 1972) and high density (Stokols, 1976) on health and cognitive performance.

There is a compelling need to determine what kinds of socioenvironmental factors may differentially predispose persons to the negative effects of the ongoing stressors and strains of daily life. To understand the forces that may increase vulnerability to daily events, we need to identify and investigate high-risk environments. The present study identified neighborhood quality, defined by the respondents' subjective evaluation of the safety and adequacy of their neighborhoods' physical and social environment, as a chronic life condition that may potentiate the relation of daily events to psychological functioning. Specifically, we examined whether the effects of daily events are more potent and longer-lasting among residents who rate their neighborhoods poorly.

\section{Major Life Events}

Major life events have been repeatedly implicated in the onset of depressive conditions, neurotic disorders, and social maladjustment (e.g., Brown \& Harris, 1978). There is also mounting evidence that stressful life events serve to precipitate and maintain physical illnesses (Cohen, 1979). Researchers, however, have seldom examined what kinds of mechanisms may link acute life stressors to health and psychological outcomes. One possibility is that major life events influence health outcomes by affecting the individual's pattern of stressful daily events and strains (e.g., Kanner et al., 1981; Pearlin, Lieberman, Menaghan, \& Mullen, 1981). Thus, daily events may be conceptualized as an intervening or mediating link between acute stressors and maladaptive behavior. In the only study that directly assessed this mediational process at the daily level, Eckenrode (1984) reported that the effects of undesirable life events on mood were mediated partly by subsequent increases in stressful daily events.

In addition to daily stressors serving to mediate the effects of major life events, life events and daily stressors may operate in a synergistic fashion; that is, with both types of stress present, the risk of impairment may be significantly greater than if only a single factor were operating. The main assumption is that individuals who have already experienced high levels of acute stressors should be less effective in coping with additional stressful events they may encounter on a daily basis. In the present study, we assessed the degree to which experience with acute life events may exacerbate the ongoing stressors and strains of daily life.

\section{Social Support}

Most people who experience psychological problems do not seek professional help. They tend, rather, to use alternative resources such as family members, friends, neighbors, employers, or community helpers (Veroff, Kulka, \& Douvan, 1981). Indeed, growing evidence that the presence of, and contact with, informal social supports may enable people to cope with stress has generated a large body of work on the adaptational significance of social network resources (Gottlieb, 1981; Mueller, 1980; Turner, 1983).

The positive functions of social resources are clearly shown in research on the connection between life's stressors and adaptation. The experience of stress, coupled with low levels of social support, has been found to be associated with psychological distress (e.g., Billings \& Moos, 1981; Gore, 1978). Other research has shown that individuals who are integrated into the social system are likely to experience a more supportive milieu during life crises and, consequently, are better able to cope with stressful events (e.g., Myers, Lindenthal, \& Pepper, 1975). Indeed, people with low levels of social support may be the ones who are most adversely affected by life changes (e.g., Dean \& Lin, 1977; Eaton, 1978).

Although it may not be possible to avoid the ongoing stressors and strains of everyday life, it is possible for individuals to mobilize support from their social network in times of perceived need. In the present study, we examined the extent to which the availability of informal social supports moderates the relation between daily events and mood.

In summary, we have emphasized three factors that may moderate the relation of daily events to mood. We expect that some factors (i.e., chronic ecological conditions, major life events) may potentiate the effects of daily events on mood, whereas others (i.e., social supports) may buffer this relation.

\section{Analytic Approaches to the Study of Daily Events}

A common approach to the analysis of daily events involves aggregating daily data over a period of many days. This approach results in an estimate of the frequency of common events experienced by the individual. For example, Kanner et al. (1981) reported that an aggregated measure of daily hassles was a significant predictor of subsequent symptom levels. Like- 
wise, Lewinsohn and Talkington (1979) reported that unpleasant daily events experienced during the previous month were related moderately to depression symptomatology. Finally, DeLongis et al. (1982) found that aggregate measures of daily hassles were better predictors of subsequent levels of physical health than were measures of life events.

On the whole, these studies suggest that aggregate measures of daily events are superior to measures of major life events in predicting average levels of mood or psychological distress. This approach, however, cannot be used to assess those aspects of daily experience associated with shifts in mood and health. To examine this issue requires longitudinal designs wherein both variables are examined over a period of many days and where the analysis retrieves the temporal relation between daily variables.

Because they preserve the temporal order of events and moods, time-series designs are appropriate for testing the expectation that daily variations in mood are determined, in part, by stressful daily experiences. A common approach for testing this expectation is demonstrated in three prospective studies of naturally occuring daily events (Lewinsohn \& Libet, 1972; Rehm, 1978; Stone, 1981). In the first step, correlation coeffcients between daily events and mood are calculated for each individual across all the observation days in the panel. In the second step, an average correlation across individuals is calculated and used to interpret the relation between daily events and mood. Results from these three daily studies show a moderate degree of convergence, despite their use of different event- and mood-assessment procedures.

But a word of caution is in order. Any advantages rendered by time-series designs can be undermined by the use of inappropriate statistical analyses, and all three of the studies noted are subject to this criticism. This point is readily demonstrated by considering the regression analog of the pooled correlation technique described earlier. In the regression case, we would estimate separate equations for each individual and then average the regression coefficients across subjects. However, specification of the regression model involves a number of assumptions regarding the error term. A critical assumption is that the covariance of any two disturbance terms is equal to zero; that is, the error terms at any one point in time are not correlated with errors at any other points. Failure to account and correct for autocorrelation can result in Type I errors in hypothesis tests because standard errors of parameter estimates are biased downward (Neter \& Wasserman, 1974). This oversight is common to the three studies already reviewed. As we will show in the present study, autocorrelation is commonly detected in time-ordered daily data, and its presence demands alternative estimation techniques. Thus, one aim of the present study was to clarify and outline appropriate statistical strategies for analyzing the relation of naturally occuring, daily events to mood.

\section{Enduring Effects of Daily Events on Mood}

Analytic difficulties notwithstanding, time-series designs furnish the data necessary to examine a host of other substantive questions. Foremost among these is the enduring influence of daily events on mood. Are shifts in mood merely a fleeting reaction to the same day's events or is one's mood affected on the days following the occurrence of a stressful event? Thus, an- other aim of the present study was to examine the enduring effects of daily events on mood.

Several studies have examined the enduring or lagged effect that daily events have on mood. Rehm (1978) found no significant lagged correlations between daily events and the next day's mood. Similarly, Lewinsohn and Libet (1972) noted that the lagged correlation between pleasant activities and mood was nonsignificant. Finally, Stone and Neale (1984), relying exclusively on the visual inspection of plots, did not detect an enduring effect of daily events on subsequent days' mood rating. Careful consideration of these studies, however, points to severe limitations.

Inspection of Lewinsohn and Libet's (1972) data reveals large individual differences in the magnitude of the correlations between mood and pleasant events; for example, intrasubject correlations of pleasant events and the next day's depressed mood ranged from -.58 to .37 . The pooled correlation reported $(-.10)$ obscures a great deal of variability in the data. In the case of Stone and Neale (1984), methodological difficulties beset their interpretation of lagged effects. To assess mood subsequent to the day with a severe event, they sampled, for each subject, an index day that was surrounded by days with no other events. This procedure eliminated $66 \%$ of the original sample. Although such selection does not appear to bias the overall contemporaneous association between events and mood, it may well have altered lagged relations and individual differences therein. Respondents included in Stone and Neale's (1984) analysis clearly experienced patterns of daily events different from those excluded on the basis of the selection procedure. Moreover, Stone and Neale's selection of a severe daily event runs counter to the theoretical impetus for examining daily stressors; it may well be the smallest and most trivial event that sends you over the edge.

In summary, the evidence to date suggests that the influence of daily events on mood does not persist beyond the day on which it occurs. However, this evidence, similar to that furnished for contemporaneous associations, derives from inappropriate estimation techniques. More important, the pooled coefficients summarized in the literature mask and misrepresent individual differences in the relation of daily events to mood and health outcomes.

\section{Method}

\section{Sample and Study Design}

The data reported in this article are from a prospective study that investigated the effects of stress and social support on the use of health services. The basic study design involved a sample of 356 women with children who were randomly selected from the population of users of a neighborhood health center in Boston. Two personal interviews were conducted 12 months apart, together with a review of the families' medical records. In addition, 96 of these women were chosen randomly and asked to complete daily diaries for 28 days immediately following the initial interviews. The results reported here are restricted to the daily diary and initial-interview data obtained from these 96 women.

The women in the sample who completed diaries were predominantly young (mean age $=33.1$ ) and had completed an average of 11 years of school. The sample represented a generally low-income population: The mean yearly income for the sample was between $\$ 7,500$ and $\$ 10,000$ in 1979. Fifty percent of the respondents were married at the time of the interviews, and $20 \%$ were Spanish speaking. No significant differences 
(at the $p<.05$ level) were observed between the diary subsample and the larger study sample on any of these characteristics.

\section{Daily Diary Measures}

The information elicited in the diaries contained two variables relevant to this study: stressful daily events and overall daily mood.

Stressful daily events. "Did anything go wrong today in the house, with the children or others in the household, at work, or elsewhere?" A dichotomous daily stress variable was constructed on the basis of responses to this question $(0=$ no stressful event; 1 = at least one stressful event). Examples of events reported include child-related (e.g., "kids got into trouble at school"), adult-related (e.g., "argued with my husband"), work-related (e.g., "troubles with my supervisor"), and nonspecific (e.g., "car wouldn't start," "lost my wallet") daily stressors. It is important to point out that the great majority of these minor stressors would not have been sampled if we had relied on the life events checklists that are often used in survey studies.

It has recently been suggested that certain reported measures of exposure to daily stressors are confounded, in part, with measures of psychological health status (Dohrenwend, Dohrenwend, Dodson, \& Shrout, 1984). In this study we explicitly sought to minimize this possible confounding by focusing on discrete events (rather than on worries or tensions) and excluding from our daily stress measure any mention of physical or psychological symptoms (e.g., fatigue, nervousness). A stressful daily event was, on average, reported on $12 \%$ of days for the 28 -day study period.

Overall daily mood. "How were your spirits today?" This meaure was accompnaied by a 7-point scale that ranged from extremely poor (1) to extremely good (7). This measure constitutes the principal outcome variable in the present analysis.

The term mood was used to refer to the generalized feeling state or overall feeling of well-being present on that particular day. The frame of reference for the diary question "How were your spirits today?" was the entire day, not a specific object or situation. It was not feasible in this community study to use more comprehensive assessments of mood states, such as the Mood Adjective Checklist (Nowlis, 1965). The term spirits was used in the wording of this question because it appeared to be less ambiguous than mood for this sample. We were primarily interested in assessing the dimension of elation versus depression, or happiness versus sadness. The term spirits has been used in previous research (e.g., Wessman, 1979) to refer to these dimensions of mood.

\section{Interview Measures}

The following measures were obtained from the initial personal interviews and represent variables that were chosen as potentially moderating the effect of daily stress on mood.

Chronic ecological stress (neighborhood quality). To investigate the potentiating effects of ecological conditions, we sought to obtain a measure that represented the ongoing and relatively unchanging (i.e., chronic) features of the respondent's life. Because our sample was drawn from an urban population of women with children, we believed that neighborhood quality was an especially critical variable to examine in relation to psychological functioning (cf. Bronfenbrenner, Moen, \& Garbarino, 1984). The neighborhoods in which these women lived were characterized by considerable variability in the quality of the housing, a diminishing supply of good rental units, rapid population turnover, and in parts of the city, a high crime rate. From the initial interviews we seleced six questions pertaining to the perceived quality of the respondent's neighborhood and housing (independent observations were

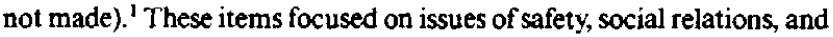
overall satisfaction. The reliability (Cronbach's alpha) of this composite scale was .61.

Major life events. In addition to measuring chronic life conditions, we sought to derive a measure of acute life changes experienced by the respondent. A count of acute life events experienced by the respondent in the 12 months preceding the interview was used as a measure of exposure to acute environmental stressors. Acute life events included experiences such as eviction, loss of job, hospitalization, and death of a family member or friend.

Social support. To investigate the stress-buffering effects of social supports, we relied on a measure of each respondent's social network in its latent form; that is, the number of persons she could rely on in times of need. The potential support measure included (a) the subset of named family members and friends she could "count" on for help with unspecified family or personal problems; (b) people she could turn to if she needed help with hypothetical problem situations (e.g., needing to borrow money); and (c) individuals she stated she could have called on, but did not, to help her cope with actual problem situations she had encountered in the previous year. Items relating more broadly to social integration (e.g., church attendance, group memberships) were not included, because we wanted a count of specifically named individuals.

\section{Data Analysis Strategy}

In this section we examine issues related to time-series analysis. Our discussion is necessarily detailed because the statistical methods involved in the present study are not yet commonly used in stress and coping research. The major issues are (a) the problem of autocorrelation and its remedies, (b) the use of pooled data, and (c) the testing of interaction effects using dummy variables.

The problem of autocorrelated errors. Given that our data involved measurements on the same individual over time, statistical issues related to repeated measurements and their associated error terms must be addressed. There are at least two generic approaches to the analysis of repeated measures. The first involves repeated measures analysis of variance or analysis of covariance (Bock, 1975), and the second involves time-series regression models (Neter \& Wasserman, 1974). We chose to use the latter because it is more readily applied to models with a large number of repeated measures.

In its simplest form (i.e., using a single independent variable) the time-series regression model can be written as

$$
Y_{t}=b_{0}+b_{1} X_{t}+e_{t}
$$

This model states that an individual's score on the dependent variable $Y$ at any time $t$ (where $t=1,2, \ldots T$ time periods) can be decomposed into the sum of a constant quantity $b_{0}$, plus a further constant $\left(b_{1}\right)$ times the individual's score on the independent variable $X$ at time $t$, and finally, a residual portion, $e_{i}$.

A crucial assumption of this model is that error terms from different time periods are unrelated. Frequently, where repeated observations are made on the same unit, this assumption is not met. One possible explanation for autocorrelated errors is the presence of omitted factors that influence the covariances among error terms. If the omitted variables follow a stable pattern from one time period to the next and cause systematic variation in the modeled behavior, temporally adjacent error terms will be correlated. Measurement error may also lead to autocorrelation if the same inaccuracies occur during successive assessments. For

\footnotetext{
${ }^{1}$ Six interview items are included in the composite measure of neighborhood quality: (a) How safe do you think your (apartment/house) is from fires? (b) How safe do you think your (apartment/house) is from break-ins and vandalism? (c) Overall, how satisfied are you with your present home? (d) How safe do you feel walking on the streets of your neighborhood after dark? (e) Sometimes neighbors help each other with things like babysitting, transportation, repairing cars, or helping repair each other's homes. Do you and those neighbors who are not your relatives do things like this often, sometimes, rarely or never? (f) Overall, how satisfied are you with this neighborhood as a place to live?
} 
example, if some component of the observed behavior is not included in successive measurements on the same individuals, positive covariances among the error terms of successive observations will result.

In theory, autocorrelation can exist between any two error terms. In practice, a frequently made assumption is that only immediately adjacent error terms are correlated to a significant degree; that is, first-order autocorrelation is assumed. This can be represented as follows,

$$
e_{t}=\rho e_{t-1}+v_{t},
$$

where $\rho$ can vary between 0 and 1 , and $v_{t}$ is free from autocorrelation. This states that an individual's error source at time $t$ is equal to that person's error score at time $t-1$ attenuated by the factor $\rho$, plus a truly random error, $v_{t}$.

Autocorrelation in a regression model does not bias the coefficients $\left(b_{0}, b_{1}\right)$; their expected value is still that found in the population. However, their standard errors are biased downwards, and hence, estimates of the influence of independent variables on the dependent variable appear to be more precise than they actually are. This can lead to the false conclusion that variables are related to one another, when in the population they are not.

Correcting for autocorrelation: Estimated generalized least squares. The method used in this study to correct for autocorrelation is estimated generalized least squares (EGLS; Judge, Griffiths, Hill, Lutkepohl, \& Lee, 1985). This procedure can be accomplished in three steps: (a) Derive an estimate of the autocorrelation coefficient $\rho$, (b) excise its influence from all components in the model, and (c) perform an ordinary least squares (OLS) regression.

In the case of Equation 1, an estimate of $\rho$ was obtained by regressing $Y_{t}$ on $X_{t}$, calculating estimates of $e_{t}$ and $e_{t-1}$, and regressing $e_{t}$ on $e_{t-1}$. This was then used to obtain a generalized difference of $Y_{t}$ as follows:

$$
Y_{t}-\hat{\rho} Y_{t-1} \text {. }
$$

Similarly expanding the right hand side of Equation 1 we obtained

$$
\begin{aligned}
Y_{t}-\hat{\rho} Y_{t-1} & =\left(b_{0}+b_{1} X_{t}+e_{t}\right)-\hat{\rho}\left(b_{0}+b_{1} X_{t-1}+e_{t-1}\right) \\
& =b_{0}(1-\hat{\rho})+b_{1}\left(X_{t}-\hat{\rho} X_{t-1}\right)+\left(e_{t}-\hat{\rho} e_{t-1}\right)
\end{aligned}
$$

An examination of Equation 3 reveals that each of the original terms in Equation 1 are present in the generalized difference form. These can be rewritten as follows:

$$
Y_{i}^{*}=b_{0} 1^{*}+b_{1} X_{i}^{*}+v_{t}
$$

Note that the use of generalized differences results in an error term, $v_{t}$, that has the randomness property required by OLS. Therefore, to correct for autocorrelation using EGLS, we constructed $Y_{t}^{*}$ and $X_{t}^{*}$ as defined earlier and used OLS regression. ${ }^{2}$

Pooling procedure. Thus far we have dealt only with the issue of intraindividual variability over time and its attendant statistical issues. However, in this study we were also concerned with individual differences. In order to examine both questions in a single model, we performed an analysis using pooled data (Judge et al., 1985).

By pooling the data of all individuals into a single matrix, it was possible to incorporate individual level, in addition to daily level, variables into the model. In this procedure, individual differences in daily relationships were assessed through the use of interaction terms (see below). Beyond those interactions that were explicitly modeled in each equation, homogeneity of regression was assumed. Consequently, pooling data resulted in a many-fold increase in degrees of freedom over single individual regression equations, thereby providing considerably more efficient parameter estimates.

Our pooling method resulted in a data matrix that had $N \times T$ rows, where $N$ is the number of cases, and $T$ is the number of repeated mea- surements per case. The structure of the data matrix was such that the first 28 rows contained all data pertaining to the first individual (where rows index days and columns index variables), the second 28 rows covered the second individual, and so on. In this matrix we included individual difference variables (e.g., neighborhood quality) as constants across days within individuals, yet variable across individuals. Thus, the first 28 observations on the individual difference variables (i.e., those relating to the first individual) took the same value, which was similarly done for each successive block of within-individual obser vations. ${ }^{3}$

The issue of autocorrelation remains as salient for pooled data as in the case where only a single set of repeated measures is used. In the present study, we estimated a single value of $\rho$ on the basis of the pooled data; that is, we assumed that $\rho$ did not vary significantly between individuals in our sample and that estimating an average $\rho$ would adequately capture the autoregressive process. ${ }^{4}$

Models incorporating individual differences. A major aim of this study was to examine sources of individual differences in the relation between stressful daily events and mood. Specifically, we were interested in whether certain variables (i.e., neighborhood quality, major life events, and social support) moderate the stress-mood relation. To test for these interaction effects, each of the moderating variables was first collapsed into three categories and then coded as two dummy $(0,1)$ variables (Cohen \& Cohen, 1975).

At this stage it is possible to specify the full statistical model we used to test hypotheses regarding individual differences in the relation of stressful daily events to mood. We do so in order to facilitate the interpretation of the regresion results and subsequent graphs. The model states that a respondent's mood on a given day is a function of her experience of a stressor on that day and her experience of a stressor on the previous day. Furthermore, it states that the effects of these variables depend on certain moderating variables. The generic interaction model is developed on the next page.

${ }^{2}$ In order to obtain a correct estimate of $b_{0}$ with the use of the OLS
procedure, it is necessary to use a column of $(1-\hat{\rho}) \mathrm{s}$ in place of the
usual column of Is (ones) in the $x$ matrix. Alternatively, the correct $b_{0}$
can be obtained by dividing the uncorrected value by $1-\hat{\rho}$. An addi-
tional adjustment is necessary for the first observation on each variable;
that is, the one for which there is no $t-1$ value, the transformed model becomes:

$$
\sqrt{1-\hat{\rho}^{2}} Y_{1}=\sqrt{1-\hat{\rho}^{2}} b_{1} X_{1}+\sqrt{1-\hat{\rho}^{2}} e_{1}
$$

This transformation approximates the generalized difference procedure in the case of the first value and preserves the full complement of data. This is especially important where $T$, the number of repeated measurements, is small.

${ }^{3}$ As outlined earlier, the regression approach to repeated measures designs incorporates individual difference variables on each person-day for each individual. Consequently, significance tests for the betweensubjects effects are based on $N \times T$ observations rather than on the appropriate $N$ observations. This results in inflated $t$ - ratios for the between-subjects parameters (cf. Cohen \& Cohen, 1975). Corrective procedures suggested for this difficulty are invalid in GLS models. However, because our substantive focus is on within-subjects effects (these include all interaction terms) the between-subjects parameters from these models were not used for inferential purposes (cf. Eckenrode, 1984). Note, however, that the point estimates for between-subjects parameters remained unbiased. In addition, the significance tests for both the within-subjects and the interaction effects were more conservative in the absence of adjustments for the between-subjects variance (cf. Cohen \& Cohen, 1975).

${ }^{4}$ It is, of course, possible to estimate $\rho$ s for each individual separately. This is not, however, a fruitful approach owing to the small number of degrees of freedom available in each instance and the resulting instability of the estimates. 
Table 1

Means, Standard Deviations, and Zero-Order Correlations for Daily Events, Daily Mood, and Moderator Variables

\begin{tabular}{lcccccc}
\hline \multicolumn{1}{c}{ Variable } & 1 & 2 & 3 & 4 & 5 & \multicolumn{1}{c}{6} \\
\hline 1. Daily events on Day $t$ & - & 0.11 & -0.23 & 0.01 & 0.00 & 0.09 \\
2. Daily events on Day $t-1$ & & - & -0.06 & 0.02 & 0.08 & 0.08 \\
3. Daily mood & & & - & -0.11 & 0.04 & -0.12 \\
4. Neighborhood quality & & & & - & -0.25 & -0.03 \\
5. Social support & & & & & - & 0.02 \\
6. Major life events & 0.12 & 0.13 & 4.90 & 12.07 & 10.57 & \multicolumn{1}{c}{4.10} \\
$\quad M$ & 0.33 & 0.33 & 1.51 & 2.85 & 3.57 & 3.02 \\
$S D$ & $0-1$ & $0-1$ & $1-7$ & $6-20$ & $3-21$ & $0-14$ \\
$\quad$ Range & & & & & & \\
\hline
\end{tabular}

Note. Minimun $N=2,229$.

Let $Y_{t}$ represent mood on day $t$ and let $S_{t}$ represent a stressful daily event on day $t$, where $S_{t}$ is 1 if an event occurs and 0 if not. Similarly, let $S_{t-1}$ stand for stressful daily event on day $t-1$, where $S_{t-1}$ equals 1 if an event occurs and 0 if not. Let $M$ represent the moderating variable, recoded to three categories: low, medium, and high. The information contained in $M$ can be captured by two dummy-coded variables $M M$ and $M H$. Let $M M$ equal 1 where an individual scores in the medium category of $M$, and 0 if not. Let $M H$ equal 1 if an individual scores in the high category of $M$ and 0 if not. It follows from these definitions that persons in the low category of $M$ must be 0 on both $M M$ and $M H$. On this basis we can express the hypothesized relation between daily events (both contemporaneous and lagged), the moderating variable, and mood as follows: ${ }^{5}$

$$
\begin{aligned}
Y_{t}=b_{0}+ & b_{1} S_{t}+b_{2} S_{t-1}+b_{3} M M+b_{4} M H+b_{5} S_{t} M M \\
& +b_{6} S_{t} M H+b_{7} S_{t-1} M M+b_{8} S_{t-1} M H+e_{t} .
\end{aligned}
$$

\section{Results}

All the results presented in this article are based on the aforementioned pooling procedure. The means, standard deviations, and intercorrelations of the variables used in the analysis are shown in Table 1. The correlations between the three moderator variables-measures of neighborhood quality, major life events, and social support-were generally low $(r \mathrm{~s}=-.03, .02$, -.25 , respectively). With the exception of neighborhood quality and social support, measures that shared $5 \%$ of their variance in common, these correlations were not significant. These findings suggest that each of the three moderator variables may capture a unique property that is, in theory, implicated in the stress process.

The zero-order correlations between the daily variables in Table 1 were not interpretable because of the presence of autocorrelation. For comparative purposes, however, it is noteworthy that the size of these coefficients was generally similar to that obtained in previous studies (cf. Lewinsohn \& Libet, 1972; Rehm, 1978; Stone, 1981).

To estimate correctly the daily stress-mood relation, we estimated a time-series regression model stating that mood on a given day is conditional on stressors of that day and stressors of the previous day. Because of the presence of significant autocorrelation $(\hat{\rho}=.51)$, this model was estimated with the use of EGLS, as outlined (for the bivariate case) in Equations 3 and 4. The results showed a significant effect of daily stress on mood $(\hat{b}=-.97, \mathrm{SE}=.07, t=-12.41, p<.0001)$. On a day characterized by at least one stressful event, mood was, on average, one scale unit lower than on a day free of such stress. The effect of the previous day's stress did not add significantly to the prediction of daily variations in $\operatorname{mood}(\hat{b}=.07, \mathrm{SE}=.07, t=-.869$, $p=.38){ }^{6}$

The preceding analysis establishes the basic relation between daily stressful events and mood changes. A major assumption of this model, however, is that the relation of stressful daily events to mood applies uniformly across individuals. A primary purpose of this study was to examine the validity of this assumption. Specifically, we examined two issues: First, under what conditions are the effects of daily stressors reduced or amplified? Second, under what conditions do the effects of daily stressors endure beyond the day of their occurrence? In the following sections we examine the contributions of three factors to the relation of stressful daily events and mood.

\section{Are the Effects of Stressful Daily Events on Mood Potentiated by Chronic Socioecologic Conditions?}

In order to demonstrate this potentiating effect we needed to show a significant interaction of daily events by neighborhood quality. The continuous measure of neighborhood quality was trichotomized and dummy coded. ${ }^{7}$ The appropriate time-series regression model assumed the form of Equation 6.

A high degree of autocorrelation $(\hat{\rho}=.49)$ required that we estimate this model by using EGLS. The results from this regression equation are presented in Table 2 . These results

\footnotetext{
s This model reduces to three submodels, one corresponding to each of the categories of the moderating variable, $M$. For those individuals low on the moderating variable, all terms containing $M M$ and $M H$ are equal to zero, and the model becomes

$$
Y_{t}=b_{0}+b_{1} S_{t}+b_{2} S_{t-1}+e_{t}
$$
}

This represents the relation between daily events and mood conditional on the presence of low $M$. Similarly, the equations for those scoring medium and high on $M$ are

$$
Y_{t}=\left(b_{0}+b_{3}\right)+\left(b_{1}+b_{5}\right) S_{t}+\left(b_{2}+b_{7}\right) S_{t-1}
$$

and

$$
Y_{t}=\left(b_{0}+b_{4}\right)+\left(b_{1}+b_{6}\right) S_{t}+\left(b_{2}+b_{8}\right) S_{t-1},
$$

respectively.

${ }^{6}$ It is possible that, in these analyses, relations observed at the daily level merely reflect individual differences. For example, the observation that stressful daily events and mood are empirically related may indicate covariation at the between-subjects (i.e., trait) level rather than unique within-subjects covariation. To assess this possibility, all models reported were reestimated with the use of individual mean-corrected daily mood scores. By subtracting each individual's mean mood score (i.e., averaged across 28 days) from that individual's 28 daily scores, all between-subjects variance was eliminated. Thus, these reestimated models unconfound intraindividual change from interindividual differences. The results from these analyses are equivalent to those reported in this study, suggesting no confounding of between- and within-subjects variance.

${ }^{7}$ The three groups were defined by $-1 / 2(S D)$ or $+1 / 2(S D)$ around the mean of the continuous measure of neighborhood quality (12.07). 
Table 2

The Effects of Contemporaneous and Lagged Daily Events on Mood: Moderating Influences of Chronic Ecological Stress (Neighborhood Quality)

\begin{tabular}{lcccc}
\hline \multicolumn{1}{c}{ Variable } & Coefficient & $S E$ & $t$ & $p$ \\
\hline$\rho^{\prime}$ & 0.49 & - & - & - \\
Intercept & 5.05 & - & - & - \\
Daily event on Day $t$ & -0.44 & .18 & -2.48 & .013 \\
Daily event on Day $t-1$ & 0.18 & .17 & 1.02 & .308 \\
Medium ES & 0.09 & .13 & 0.71 & .478 \\
High ES & -0.31 & .16 & -1.95 & .052 \\
DE $\times$ Medium ES & -0.61 & .20 & -2.98 & .003 \\
DE $_{t} \times$ High ES & -0.77 & .24 & -3.15 & .002 \\
DE $_{t \rightarrow 1} \times$ Medium ES & -0.18 & .20 & -0.88 & .377 \\
DE $_{t \rightarrow 1} \times$ High ES & -0.64 & .24 & -2.69 & .007 \\
\hline
\end{tabular}

Note. The coefficients in this table are unstandardized regression weights. $\mathrm{DE}=$ daily event; $\mathrm{ES}=$ ecological stress. $R^{2}=.08$.

autocorrelation coefficient.

showed significant interaction effects of chronic stress, as indexed by neighborhood quality, with the same day's stressful events $(\hat{b}=-.61, p<.003 ; \hat{b}=-.77, p<.002)$, as well as with the previous day's stressful events $(\hat{b}=-.64, p<.007)$. These findings confirmed our expectation that the effect of daily events on mood is conditioned by levels of chronic stress.

The full model was decomposed in order to illustrate significant differences across levels of chronic stress (see Equations 79). Figure 1 shows the effect of daily stress on the same day's mood for women in varying conditions of low-, medium-, and high-chronic stress by plotting the regression lines in each of these groups. These findings suggest that the effect of stressful daily events on the same day's mood increases monotonically as a function of chronic stress. The influence of daily events on mood was potentiated even at moderate levels of chronic stress. Among women in this middle group, the coefficient for daily stress $(\hat{b}=-1.04)$ was more than twice the size of the corre-

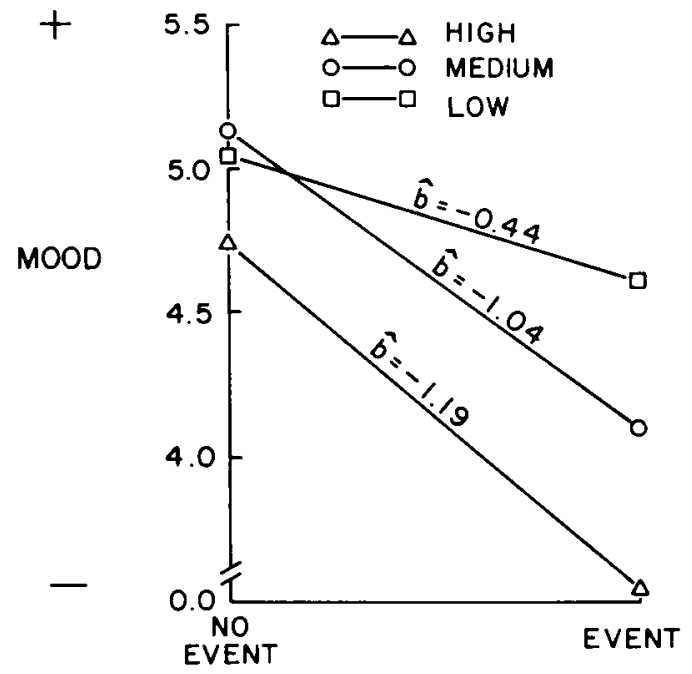

Figure 1 . The effect of contemporaneous daily events on daily mood by neighborhood quality, controlling for lagged daily events.

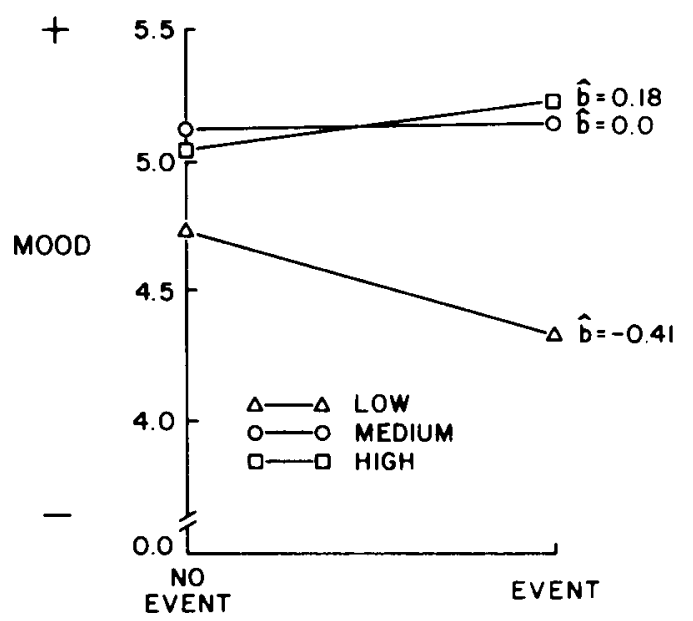

Figure 2. The effect of lagged daily events on daily mood by chronic ecological stress, controlling for contemporaneous daily events.

sponding coefficient for women in low-chronic stress conditions $(\hat{b}=-.44)$. The negative effect of stressful daily events was most pronounced among women in high-chronic stress conditions. In this group, the coefficient for daily stress on mood $(\hat{b}=-1.19)$ was nearly 3 times the size of its effect in the lowchronic stress group $(\hat{b}=-.44)$.

Figure 2 shows the effect of the previous day's stressful event on mood. As expected, the effect of stressful daily events lagged one day tapered off substantially. But this process was not uniform across conditions of chronic stress. Among women in lowand moderate-chronic stress conditions, the effect of the previous day's stressful event was negligible. Among women in high-chronic stress conditions, the effect of the previous day's stressful event on mood was quite substantial $(\hat{b}=-.41)$ and differed significantly from the relations observed in the two remaining groups.

In summary, these results suggest that the adverse effect of stressful daily events on the same day's mood rating increased as a function of chronic stress conditions (see Figure 1). But the effect of the previous day's stressful event endured only among women who rated their neighborhoods as being of poor quality (see Figure 2). Women in conditions of moderate ecologic stress fared no worse than their counterparts in conditions of relatively low-chronic stress.

\section{Are the Negative Effects of Stressful Daily Events Exacerbated by Major Life Events?}

As in the previous model, a three-category coding procedure was applied to the continuous measure of stressful life events. ${ }^{8}$ A high degree of autocorrelation $(\hat{\rho}=.50)$ required estimating the model with the use of EGLS.

The results from this regression equation are shown in Table 3. As expected, the point estimates for the main effects terms for major life events showed that, in the absence of stressful

\footnotetext{
${ }^{B}$ The three groups were defined by $-1 / 2(S D)$ or $+1 / 2(S D)$ around the mean of the continuous measure of the number of acute life events $(4.10)$.
} 
Table 3

The Effects of Contemporaneous and Lagged Daily Events on Mood: Moderating Influences of Major Life Events

\begin{tabular}{lcccc}
\hline \multicolumn{1}{c}{ Variable } & Coefficient & $S E$ & $t$ & $p$ \\
\hline$\rho^{\mathrm{a}}$ & 0.50 & - & - & - \\
Intercept & 5.28 & - & - & - \\
DE on Day $t$ & -1.50 & .20 & -7.63 & .000 \\
DE on Day $t-1$ & 0.00 & .19 & 0.01 & .995 \\
Medium no. LEs & -0.30 & .13 & -2.34 & .019 \\
High no. LEs & -0.39 & .16 & -2.43 & .015 \\
DE $_{\mathrm{t}} \times$ Medium LE & 0.60 & .22 & 2.69 & .007 \\
$\mathrm{DE}_{\tau} \times$ High LE & 0.73 & .25 & 2.95 & .003 \\
$\mathrm{DE}_{\mathrm{t}-1} \times$ Medium LE & -0.25 & .22 & -1.14 & .254 \\
$\mathrm{DE}_{\mathrm{\tau}-1} \times$ High LE & 0.27 & .24 & 1.13 & .259 \\
\hline
\end{tabular}

Note. $\mathrm{DE}=$ daily event. $\mathrm{SS}=$ social support. $\mathrm{LE}=$ life event. $R^{2}=.08$.

"Autocorrelation coefficient.

daily events, the effect of major stressors was distinctly negative $(\hat{b}=-.30 ; \hat{b}=-.39)$. This is shown graphically by intercept differences in Figure 3. The findings also showed significant interactions between levels of major life events and stressful daily events $(\hat{b}=.60, p<.007 ; \hat{b}=.73, p<.003)$. These results are presented in Figure 3.

Contrary to expectations, the effect of stressful daily events on mood was not exacerbated by major life events; it was actually minimized in the presence of such events. As we moved across the sample from those women who had experienced relatively few or no life events to those who had experienced a moderate or large number of such events, the effect of stressful daily events became significantly less negative $(\hat{b}=-1.50$ vs. -.90 and -.77$)$. In the case of lagged daily stressors, intercept differences by acute life events showed the predicted pattern; a greater number of major stressors resulted in depressed mood. However, there were no significant slope differences between the three major stressor groups.

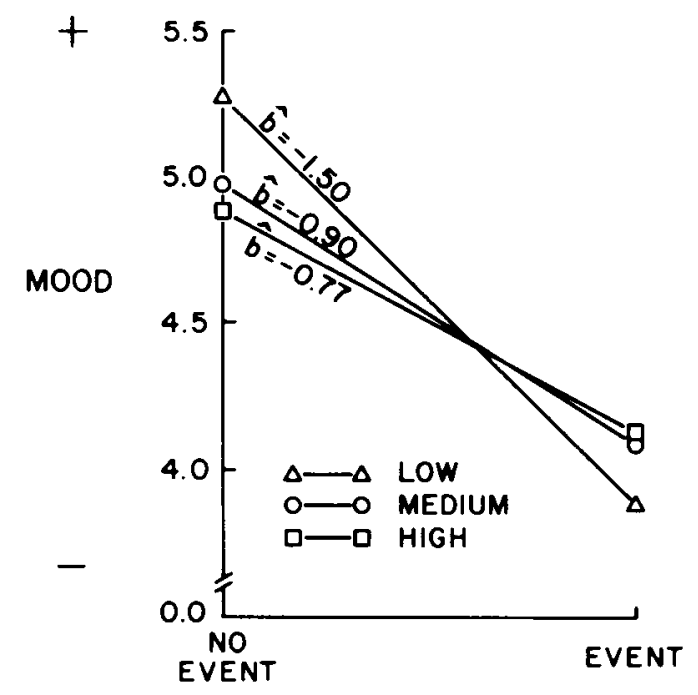

Figure 3. The effect of contemporaneous daily events on daily mood by number of major life events, controlling for lagged daily events.
Table 4

The Effects of Contemporaneous and Lagged Daily Events: Moderating Influences of Social Support

\begin{tabular}{lcccc}
\hline & Coefficient & $S E$ & $t$ & $p$ \\
\hline$\rho^{\mathrm{a}}$ & 0.51 & & & \\
Intercept & 4.92 & & & \\
DE on Day $t$ & -1.12 & .16 & -7.02 & .000 \\
DE on Day $t-1$ & -0.36 & .17 & -2.28 & .023 \\
Medium SS & 0.11 & .12 & 0.93 & .350 \\
High SS & 0.21 & .14 & 1.51 & .131 \\
DE $_{t} \times$ Medium SS & 0.17 & .18 & 0.87 & .385 \\
DE $_{t} \times$ High SS & 0.25 & .19 & 1.28 & .202 \\
DE $_{t-1} \times$ Medium SS & 0.23 & .21 & 1.08 & .280 \\
DE $_{t-1} \times$ High SS & 0.56 & .21 & 2.67 & .007 \\
\hline
\end{tabular}

Note. $\mathrm{DE}=$ daily event. $\mathrm{SS}=$ social support. $R^{2}=.07$.

Autocorrelation coefficient.

\section{Are the Effects of Stressful Daily Events Buffered by Social Support?}

As with the previous two models, a categorical coding procedure was applied to the continuous measure of social support in order to test for Stress $\times$ Social Support interaction effects. ${ }^{9}$ Estimates of the autocorrelation coefficient showed a significant correlation between consecutive error terms $(\hat{\rho}=.51)$, and the EGLS procedure was used to estimate the model. The results of this regression equation are presented in Table 4 . There were no significant interaction effects of daily stress by social support. These findings suggest that the amount of potential supporters available in time of need does not alter the effect of stressful daily events on the current day's mood.

The results, however, showed a significant interaction of the previous day's stressful event with the number of potential supporters $(\hat{b}=.56, p<.007)$. This effect is shown in Figure 4 . These findings suggest that the buffering effect of social supports on the enduring influence of stressful daily events increased by regular amounts as we moved from conditions of low and moderate support to those of high support. In conditions of low support, the enduring effect of stressful events was maximal $(\hat{b}=-.36)$. Under conditions of moderate support, the enduring effect of daily events was minimized $(\hat{b}=-.12)$. In this case, the negative influence of the previous day's stressful event was reduced by two thirds of its original effect. Finally, under conditions of high support, a complete shift occurred. In this last group, the effect of the previous day's stressful event was positive in relation to $\operatorname{mood}(\hat{b}=.20)$.

In summary, these results indicate that social support does not buffer the effect of stressful daily events on the same day's mood. However, social support is critical in relation to the enduring effect of stressful daily events. Under conditions of low support, the adverse effects of daily stressors persist beyond the day of their occurrence. In contrast, under conditions of high support, stressful daily events actually increase the likelihood of a positive mood on the following day.

\footnotetext{
${ }^{9}$ The three groups were defined by $-1 / 2(S D)$ or $+1 / 2(S D)$ around the mean of the continuous measure of potential social supports (10.57).
} 


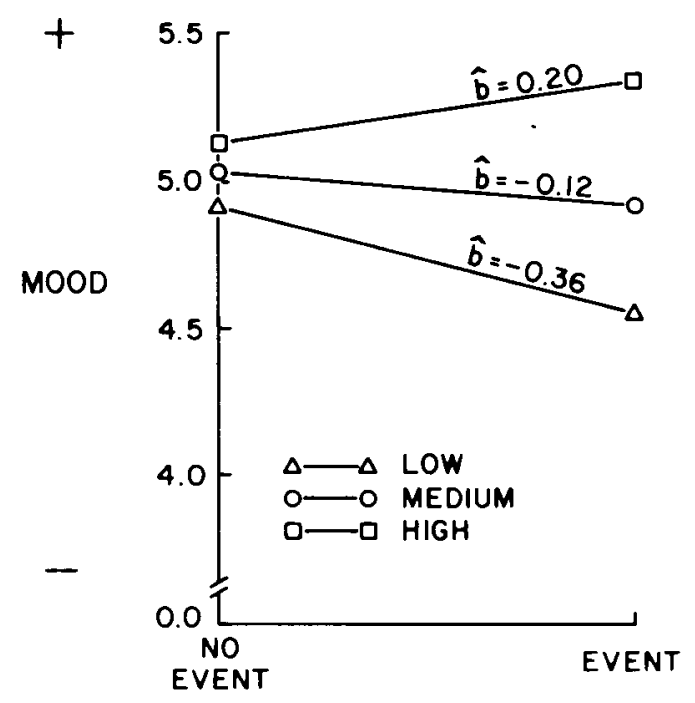

Figure 4. The effect of lagged daily events on daily mood by level of social support, controlling for contemporaneous daily events.

\section{Discussion}

A primary task for research on psychological distress is to determine the difference in vulnerability to stressful conditions of different individuals within society. We have approached this task by examining three factors-chronic ecological conditions, major life events, and social supports-that may potentiate or buffer the effects of stressful daily events on mood. Our results suggest that stressful daily events have different effects on individuals if they occur under different conditions. In the following section we discuss the mechanisms that may moderate the stress-mood relation. In the final section we return to assess the usefulness of our approach to the daily study of the stress process.

\section{Chronic Socioecologic Conditions}

The search for conditions that affect psychological functioning inevitably leads to questions about the ecological circumstances of people's lives. Research has repeatedly implicated ecological conditions in the onset of psychological distress (e.g., Rutter \& Quinton, 1977). This is also clearly borne out in our analysis of neighborhood quality. As we move from relatively safe neighborhoods to high-risk neighborhoods, the immediate effects of stressful daily events become increasingly more adverse. What accounts for the potentiating effects of these chronic conditions on the relation of daily events to mood? One possibility is that experiences with chronically stressful conditions may have heightened these women's level of arousal and anxiety and, consequently, impeded their effective action.

Consider the hypothetical case of a woman whose apartment in dilapitated row house is vulnerable to fire, who dreads a possible mugging, and who fears for the safety of her children at play in a congested street (all women in the sample had a least one dependent child at home). The behavioral consequences of such chronic tension may result in heightened reactivity to minor events and reduce her ability to manage effectively even minor disruptions in her daily routine. In this respect, it is note- worthy that the effects of stressful daily events on mood endure only among women who reside in neighborhoods of the poorest perceived quality.

We have also considered the possibility that the potentiating effects of neighborhood quality simply reflect class differences between women in our sample. But this is unlikely to be the case because our sample is drawn from a generally low-income population. For example, the correlation between income level and neighborhood quality was -.14 , a surprisingly low-order coefficient that most likely reflects the restricted range of income level in this sample. As such, neighborhood quality may be regarded as a vulnerability factor that further differentiated women who, given their low-income levels and social status, may be already at risk. Moreover, our use of perceptual indicators of neighborhood quality highlights the benefits accrued from proximal approaches to environmental assessment (cf. Jessor, 1981). Such approaches may provide a more precise understanding of the immediate impact of the environment than do distal indicators such as social class.

We believe that underlying the potentiating effects of chronic socioecologic conditions is a process of heightened reactivity. Moreover, we have argued that these potentiating effects are attributable to conditions of living in poor-quality neighborhoods. But it is also possible that constitutionally frail individuals are more likely to live in conditions of chronic stress. In this respect, the selection of individuals with relatively ineffective coping skills and resources into poor-quality neighborhoods may play an equally important role in accounting for their heightened reactivity to stressful daily events. This alternative explanation underscores the need to consider both social-causation and social-selection perspectives in disentangling the relation of social structure to health (e.g., Kessler, 1979; Kessler \& Cleary, 1980; Kohn \& Schooler, 1983; Wheaton, 1978). The critical difference between these two contending perspectives lies in the locus of causation. Note, however, that both explanations are consistent with a process of heightened emotional reactivity. The present findings support the premise that to understand the stress process we must locate individuals in their particular environments; how individuals get there is a separate issue to be addressed in future research.

\section{Major Life Events}

We expected acute life stressors occurring in the 12 months prior to the diary period to exacerbate the effect of stressful daily events on mood. Contrary to expectations, acute life stressors actually diminished the effects of daily events on mood. The experience of a minor daily event was less influential in the presence of a large number of acute life stressors than in the presence of few or no acute life stressors. What accounts for this paradoxical finding?

An analogy to this problem may be found in classic psychophysics and methods for the study of individual threshold values (Stevens, 1974). We refer to Fechner's Law: The greater the weight of a standard, the greater must be the difference between the standard and a comparison weight before any noticeable difference can be detected. Thus, if you are holding a three pound package, the addition of another pound will certainly be detected. But the addition of another pound to a $100-1 b$ package will most likely go unobserved. Now imagine that in the recent 
past you have been evicted from your apartment, fired from your job, and hospitalized for a major illness. A flat tire may have little additional influence on your mood. But in the absence of such acute life changes, a flat tire will most likely ruin your day. Thus, under conditions of acute stress, the capacity of minor stressors to produce a detectable response may be lessened. In contrast, when adaptations to acute life events are not required, the responsivity threshold to minor stressors decreases, and their effect on mood is substantially increased. This explanation, of course, rests on the assumption that the greater the number of acute stressors, the greater the adaptational load on the individual; an assumption underlying most life events research (e.g., Holmes \& Masuda, 1974).

A process similar to that argued here has been hinted at in previous work. Dohrenwend and Dohrenwend (1978) emphasized the importance of a previous history of stressors in dealing with current crises. They suggested that prior exposure to stressors may lead to habituation so that later experiences with stressors may actually result in lower levels of emotionality. No empirical studies, however, have examined interactions between various types of stressors. In testing for such interactions, the present study raises the intriguing possibility of a threshold effect in sensitivity to stress.

Another possible explanation for these effects centers on the learning of coping skills. It may be that experience in coping with major life events provides the individual with feedback on effective and ineffective ways to cope with stress. Such experiential learning may then carry over to subsequent stressors and coping strategies on the daily level. Although plausible for some individuals, this explanation would require direct evidence for the application of particular coping strategies from major events to minor daily events, data not available in the present study.

\section{Social Supports}

Stress researchers have increasingly focused on the importance of social networks and close personal relationships in buffering the effects of stressful events (e.g., Gottlieb, 1981). Stress buffering is generally equated with the presence of a significant Stress $\times$ Social Support interaction term in the analysis; that is, the relation of stress to psychological functioning is contingent on levels of available social supports.

Consistent with previous research with major life events (e.g., Gore, 1978), the present findings show that social supports moderate the effects of stressful daily events on self-reported mood. More importantly, our findings suggest that the moderating effect of social supports occurs only on the day following the occurrence of a stressful event. These results suggest that social supports are most effective in buffering the enduring effects of stressful daily events. Several explanations may account for the lagged-moderating effect of social supports on the daily stressmood relation.

The first explanation centers on the mobilization of social supports to cope with daily crises. The presence of supportive relationships in an individual's social network indicates a buffering potential; it does not imply that social support is always and immediately available. Thus, it is possible that the observed one-day lag reflects the amount of time needed to mobilize support from a network of social ties. This finding highlights the need for research on the mobilization of social supports (e.g., Eckenrode, 1983). In this regard, future research will need to examine the conditions under which support is mobilized, the means undertaken to mobilize support, as well as the onset and duration of the mobilization process.

A second explanation centers on the timing of the influence of social supports. Although social supports may be mobilized immediately, it is possible that the benefits of such supports are reaped only later. Thus, supportive relationships may not ensure against the immmediate adverse effects of stressful daily events, but they may keep any enduring debilitation in check.

Turning to the pattern of lagged effects, we find that stressful daily events have a positive influence on mood given a large number of potential supporters. Social supports not only buffer the effects of stressful events; under some conditions stressful events, in combination with social supports, actually enhance positive mood. All too often we assume that stressful experiences must result in debilitation and decline. Such outcomes are common enough. But given appropriate conditions, experience with problem situations and stressful events may also pave the road for psychological growth and well-being, perhaps by providing the context for positive social exchange among network members. In addition to the findings reported here, evidence for this process has emerged from longitudinal studies of drastic life change. For example, Elder and his colleagues (Caspi \& Elder, 1986; Elder, Liker, \& Jaworski, 1984) have shown that deprivation experiences in the Great Depression were often associated with negative outcomes for working-class women. However, among middle-class women, who often possessed the skills and resources necessary to master stressful experiences, the long-term effects of the Great Depression were actually positive, as reflected in their greater emotional stability, self-confidence, and life satisfaction. Such findings provide compelling empirical evidence for the observation that the combination of maximum demand with maximum support often provides for the most healthful outcomes (Bronfenbrenner et al., 1984). ${ }^{10}$

\section{Conclusion}

A critical question for research on the stress process concerns the nature of those variables that determine which individuals are likely to be affected by stressful events and which are likely to be spared its effects. What factors potentiate the effects of stressful events on psychological functioning? Conversely, we may ask what are the protective or ameliorating circumstances? To address these questions research on the stress process must be formulated with explicit reference to individual differences.

In the present study we have examined the effects of stressful daily events on mood as a function of both vulnerability in the individual and provocativeness in the environment. The findings clearly indicate that the effects of daily events are not uniform; under certain conditions they are distinctly negative; under others they are actually positive. Significant interaction

\footnotetext{
${ }^{10}$ Bronfenbrenner attributed this observation to the Soviet social psychologist A. S. Makarenko. In describing the success of "colonies" conceived for the rehabilitation of homeless children and youth following the civil wars in the Soviet Union, Makarenko offered the formulation "the maximum of demand with the maximum of support" as an essential condition for facilitating psychological growth.
} 
effects suggest that the underlying process that governs the relation between any two variables is not uniform across individuals. Interaction effects, however, cannot identify what process, per se, is operating. Indeed, like other research in uncharted territory, the present findings have resulted in more questions than answers. These findings clearly await replication and, moreover, suggest a critical research agenda. Finding statistical interaction effects in stress research should be viewed as the starting point for future studies designed to test hypotheses regarding process (cf. Rutter, 1983). To this end, the present study has made several contributions.

First, we have shown that the daily stress process is conditioned by specific ecological factors (e.g., neighborhood quality), as well as individual histories (e.g., experience with a series of acute life events). These findings underscore the usefulness of research on stress that is formulated with explicit reference to potentiating and protective factors. In recent years, mental health researchers have begun to examine protective factorsthose attributes of individuals or environments that provide resistance to risk and foster patterns of adaptation-in addition to potentiating factors, those that trigger a breakdown. We believe that core concepts of resilience and vulnerability will acquire true meaning only in an interactive sense; neither is a property of the individual or environment, per se. Indeed, as the present findings suggest, for any particular person, psychological reactivity is determined by vulnerability to the condition at hand and by the intensity of the environmental provocation.

Second, the temporal framework adopted in the present study may yield the type of data that are most promising for testing hypotheses about the stress process. Most models of stress assume implicitly that stressful events result in alterations of daily routines. Thus, it is precisely at the daily level, where ordinary routines take place, that we may best understand how individuals cope with and manage such crises.

Because the daily framework preserves the temporal relation among variables, testing for covariation within individuals and across time is a more powerful approach to testing cause-effect relations in nonexperimental data than the more restrictive between-subjects designs. Moreover, by observing different patterns of covariation within individuals across time, we may begin to identify the individual attributes and environmental circumstances that are most critical in the stress process. The benefits of such approaches are also evident in research on stress in families. Patterson (1983) has argued that environmental stressors influence individual functioning by altering interactional processes between family members. As his work suggests, such alterations and their consequences are best understood in the context of daily patterns of family transactions.

Finally, to examine the temporal relation of stressful daily events to mood, we have used statistical tools that can be applied readily and effectively to the analysis of the stress process. Time-series models are used extensively by economists in the analysis of aggregate data. However, these models have not been applied frequently to the analysis of individual behavior (but see Gottman, 1981). Time-series models make full use of the information contained in time-ordered data, and individualdifference hypotheses can be easily incorporated in their estimation. We believe the procedures adopted in this study will prove valuable in research on the stress process and offer an alternative to the common practice of aggregating successive observa- tions. In recent years, this latter approach has been advocated in the name of demonstrating consistency in behavior (e.g., Epstein, 1979). Toward this research aim, aggregating daily data may result in elegant demonstrations of consistency, but this approach also results in the loss of process-oriented information and, as the present study suggests, obscures critical variations in the relation between variables over time.

Of course, neither shifting the level of analysis to the daily level nor adding tools to our statistical arsenal will, by themselves, result in a useful general theory of stress. We do believe, however, that the analytic approaches advanced in this study, coupled with theoretical orientations linking person and context, may result in a clearer specification of the stress process and enhance our ability to differentiate vulnerable individuals from resilient ones.

\section{References}

Billings, A., \& Moos, R. H. (1981). The role of coping responses and social resources in attenuating the stress of life events. Journal of Behavioral Medicine, 4, 139-157.

Bock, D. (1975). Multivariate statistical methods in behavioral research. New York: McGraw-Hill.

Bronfenbrenner, U., Moen, P., \& Garbarino, J. (1984). Child, family and community. In R. Parke, R. Emde, H. McAdoo, \& E. Sackett (Eds.), Review of child development research (Vol. 7, pp. 283-328). Chicago: University of Chicago Press.

Brown, G. W., \& Harris, T. (1978). Social origins of depression: A study of psychiatric disorder in women. New York: Free Press.

Caspi, A., \& Elder, G. H., Jr. (1986). Life satisfaction in old age: Linking social psychology and history. Psychology and Aging, 1, 18-26.

Cohen, F. (1979). Personality, stress, and the development of physical illness. In G. C. Stone, F. Cohen, \& N. E. Adler (Eds.), Health psychol$o g y$ (pp. 77-111). San Francisco: Jossey-Bass.

Cohen, J., \& Cohen, P. (1975). Applied multiple regression/correlation analysis for the behavioral sciences. Hillsdale, $\mathrm{NJ}$ : Erlbaum.

Dean, A., \& Lin, N. (1977). The stress-buffering role of social support. Journal of Nervous and Mental Disease, 165, 403-417.

DeLongis, A., Coyne, J. C., Dakof, G., Folkman, S., \& Lazarus, R. S. (1982). Relationship of daily hassles, uplifts, and major life events to health status. Health Psychology, 1, 119-136.

Dohrenwend, B. S., \& Dohrenwend, B. P. (1978). Some issues in research on stressful life events. Journal of Nervous and Mental Disease, I66, 7-15.

Dohrenwend, B. S., Dohrenwend, B. P., Dodson, M., \& Shrout, P. E. (1984). Symptoms, hassles, social supports, and life events: Problems of confounded measurements. Journal of Abnormal Psychology, 93, 222-230.

Eaton, W. W. (1978). Life events, social supports, and psychiatric symptoms: A reanalysis of the New Haven data. Journal of Health and Social Behavior, 19, 230-234.

Eckenrode, J. (1983). The mobilization of social supports: Some individual constraints. American Journal of Community Psychology, 11 . 509-527.

Eckenrode, J. (1984). The impact of chronic and acute stressors on daily reports of mood. Journal of Personality and Social Psychology. 46 . 907-918.

Elder, G. H., Jr., Caspi, A., \& Van Nguyen, T. (1986). Resourceful and vulnerable children: Family influences in stressful times. In R. K. Silbereisen, K. Eyferth, \& G. Rudinger (Eds.), Development as action in context: Problem behavior and normal youth development ( $\mathrm{pp}$. 167-187). New York: Springer.

Elder, G. H., Jr., Liker, J., \& Jaworski, B. J. (1984). Hardship in lives: Depression influences from the 1930's to old age in postwar America. 
In K. McCluskey \& H. W. Reese (Eds.), Life-span developmental psychology: Historical and cohort effects (pp. 161-201). New York: Academic Press.

Epstein, S. (1979). The stability of behavior: 1. On predicting most of the people much of the time. Journal of Personality and Social Psychology, 37, 1097-1126.

Garbarino, J., \& Sherman, D. (1980). High-risk neighborhoods and high-risk families: The human ecology of child maltreatment. Child Development, 51, 188-198.

Glass, D. C., \& Singer, J. E. (1972). Urban stress. New York: Academic Press.

Gore, S. (1978). The effect of social support in moderating the health consequences of unemployment. Journal of Health and Social Behavior, 19, 157-165.

Gottlieb, B. (Ed.) (1981). Social networks and social support. Beverly Hills, CA: Sage.

Gottman, J. M. (1981). Time series analysis for the behavioral sciences. Cambridge, England: Cambridge University Press.

Holmes, T. H., \& Masuda, M. (1974). Life change and illness susceptability. In B. S. Dohrenwend \& B. P. Dohrenwend (Eds.), Stressful life events: Their nature and effects (pp. 45-72). New York: Wiley.

Jessor, R. (1981). The perceived environment in psychological theory and research. In D. Magnusson (Ed.), Toward a psychology of situations: An interactional perspective (pp. 297-317). Hillside, NJ: Erlbaum.

Judge, G. G., Griffiths, W. E., Hill, R. C., Lutkepohl, H., \& Lee, T. C. (1985). The theory and practice of econometrics. New York: Wiley.

Kanner, A. D., Coyne, J. C., Schaefer, C., \& Lazarus, R. S. (1981). Comparison of two modes of stress measurement: Daily hassles and uplifts versus major life events. Journal of Behavioral Medicine, 4, 1-39.

Kessler, R. (1979). Stress, social status, and psychological distress. Journal of Health and Social Behavior, 20, 259-272.

Kessler, R., \& Cleary, P. (1980). Social class and psychological distress. American Sociological Review, 45, 463-478.

Kohn, M. L., \& Schooler, C. (1983). Work and personality: An inquiry into the impact of social stratification. Norwood, NJ: Ablex.

Lazarus, R. S., \& Cohen, J. B. (1977). Environmental stress. In I. Altman \& J. Wohlwill (Eds.), Human behavior and environment: $A d$ vances in theory and research (Vol. 2, pp. 89-127). New York: Plenum Press.

Leighton, A. (1974). Social disintegration and mental disorder. In G. Caplan (Ed.), American handbook of psychiatry (Vol. 2, pp. 411423). New York: Basic Books.

Lewinsohn, P. M., \& Libet, J. (1972). Pleasant events, activity schedules, and depression. Journal of Abnormal Psychology, 79, 291-295.

Lewinsohn, P. M., \& Talkington, J. (1979). Studies on the measurement of unpleasant events and relations with depression. Applied Psychological Measurement, 3, 83-101.

McLean, P. D. (1976). Depression as a specific response to stress. In I. G. Sarason \& C. D. Spielberger (Eds.), Stress and anxiey (Vol. 3, pp. 297-323). Washington, DC: Hemisphere.

Mueller, D. P. (1980). Social networks: A promising direction for research on the relationship of the social environment to psychiatric disorder. Social Science and Medicine, 14, 147-161.

Myers, J. K., Lindenthal, J. J., \& Pepper, M. P. (1975). Life events, social integration, and psychiatric symptomatology. Journal of Health and Social Behavior, 16, 421-427.
Neter, J., \& Wasserman, W. (1974). Applied linear statistical models. Homewood, IL: Irwin.

Neugebauer, R. (1981). The reliability of life-event reports. In B. S. Dohrenwend \& B. P. Dohrenwend (Eds.), Stressful life events and their contexts (pp. 85-107). New Brunswick, NJ: Rutgers University Press.

Nowlis, V. (1965). Research with the Mood Adjective Checklist. In S. S. Tomkins \& C. E. Izard (Eds.), Affect, cognition and personality (pp. 352-389). New York: Springer.

Patterson, G. R. (1983). Stress: A change agent for family process. In N. Garmezy \& M. Rutter (Eds.), Stress, coping and development in children (pp. 235-264). New York: McGraw-Hill.

Pearlin, L. I., Lieberman, M. A., Menaghan, E., \& Mullen, J. T. (1981). The stress process. Journal of Health and Social Behavior, 22, 337356.

Rabkin, J. G., \& Struening, E. L. (1976). Life events, stress, and illness. Science, 194, 1013-1020.

Rehm, L. P. (1978). Mood, pleasant events, and unpleasant events: Two pilot studies. Journal of Consulting and Clinical Psychology, 46, 854859.

Rutter, M. (1983). Statistical and personal interactions: Facets and perspectives. In D. Magnusson \& V. Allen (Eds.), Human development: An interactional perspective (pp. 295-320). New York: Academic Press.

Rutter, M., \& Madge, N. (1976). Cycles of disadvantage. London: Heinemann.

Rutter, M., \& Quinton, D. (1977). Psychiatric disorder: Ecological factors and concepts of causation. In H. McGurk (Ed.), Ecological factors in human development (pp. 173-187). Amsterdam: North-Holland.

Srole, L., Langner, T. S., Michael, S. T., Oples, M. K., \& Rennie, T. A. C. (1962). Mental health in the metropolis. New York: McGrawHill.

Stevens, S. S. (1974). Psychophysics. New York: Wiley.

Stokols, D. (1976). The experience of crowding in primary and secondary environments. Environment and Behavior, 8, 49-86.

Stone, A. A. (1981). The association between perceptions of daily experiences and self- and spouse-related moods. Journal of Research in Personality, 15, 510-522.

Stone, A. A., \& Neale, J. M. (1984). Effects of severe daily events on mood. Journal of Personality and Social Psychology, 46, 137-144.

Thoits, P. A. (1981). Undesirable life events and psychophysiological distress: A problem of operational confounding. American Sociological Review, 46, 97-109.

Turner, R. J. (1983). Direct, indirect, and moderating effects of social support on psychological distress and associated conditions. In H. B. Kaplan (Ed.), Psychosocial stress (pp. 105-155). New York: Academic Press.

Veroff, J., Kulka, R. A., \& Douvan, E. (1981). Mental health in America. New York: Basic Books.

Wessman, A. E. (1979). Moods: Their personal dynamics and signifcance. In C. E. Izard (Ed.), Emotions in personality and psychopathology (pp. 73-102). New York: Plenum Press.

Wheaton, B. (1978). The sociogenesis of psychological disorder: Reexamining the causal issues with longitudinal data. American Sociological Review, 43, 383-403.

Received August 13, 1984

Revision received March 4, 1985 